\title{
Thermal Emission from Transiting Very-Hot Jupiters: Prospects for Ground-based Detection at Optical Wavelengths
}

\author{
Mercedes López-Morales ${ }^{1} \&$ Sara Seager ${ }^{2}$ \\ mercedes@dtm.ciw.edu, seager@mit.edu
}

\begin{abstract}
Very hot Jupiters (VHJs) are defined as Jupiter-mass extrasolar planets with orbital periods shorter than three days. For low albedos the effective temperatures of irradiated VHJs can reach 2500-3000 K. Thermal emission from VHJs is therefore potentially strong at optical wavelengths. We explore the prospects of detecting optical-wavelength thermal emission during secondary eclipse with existing ground-based telescopes. We show that OGLE-TR-56b and OGLE-TR$132 \mathrm{~b}$ are the best suited candidates for detection, and that the prospects are highest around $z^{\prime}$-band $(\sim 0.9 \mu \mathrm{m})$. We also speculate that any newly discovered VHJs with the right combination of orbital separation and host star parameters could be thermally detected in the optical. The lack of detections would still provide constraints on the planetary albedos and re-radiation factors.
\end{abstract}

Subject headings: binaries:eclipsing — planetary systems — stars:individual (OGLETR-56, OGLE-TR-132) — techniques: photometric

\section{Introduction}

Five hot Jupiters have published lower atmosphere measurements (Charbonneau et al. 2002, 2005; Deming et al. 2005, 2006; Harrington et al. 2006; Knutson et al. 2007; Grillmair et al. 2007; Richardson et al. 2007; Tinetti et al. 2007). Several upper atmosphere detections have also been reported (Vidal-Madjar 2003, 2004; Ballester et al. 2007). All these measurements have been made from space, with the Spitzer and Hubble telescopes. Exoplanet atmosphere detection from the ground has so far been elusive, despite many tries

\footnotetext{
${ }^{1}$ Carnegie Fellow. Carnegie Institution of Washington, Department of Terrestrial Magnetism, 5241 Broad Branch Rd. NW, Washington D.C., 20015, USA

${ }^{2}$ Department of Earth, Atmospheric, and Planetary Sciences, Department of Physics, Massachusetts Institute of Technology, 77 Massachusetts Ave., Cambridge, MA 02139-4307 USA
} 
(e.g. Deming et al. 2007; Snellen \& Covino 2007; Snellen 2005). Yet, ground-based detection capability would greatly facilitate studies of hot Jupiter atmospheres.

Hot Jupiters are Jupiter-mass planets in 3 to 9 day period orbits. Very hot Jupiters (VHJs) are those in 1 to 3 day period orbits. These planets are heated primarily from incident radiation from their parent stars. The closer the planet is to the star, the hotter the planet will be. VHJs orbiting solar-type stars may have effective temperatures, $T_{p}$, as high as 2500-3000K. Therefore, VHJs may be as hot as brown dwarfs or very low-mass stars. In this case, the contribution of the thermal emission from the planet to the total light of the planet-star system will be significantly high, and could be detected in the optical or near-infrared during secondary transits.

Eleven transiting VHJs are currently known (see Table 1). We explore the thermal radiation contribution of these planets at wavelengths between $\mathrm{V}$ and K-band (0.5-2.5 $\mu \mathrm{m})$, and the prospects of detecting their secondary transits with current ground-based instruments. Our study focuses on the thermal emission from the planets' lower atmosphere, defined to be in the 1 to $10^{-5}$ bar pressure range.

\section{Thermal Emission vs. Reflected Light}

For VHJs at optical wavelengths one usually thinks of the reflected light without considering that, depending on the VHJ effective temperature, thermal emission could dominate reflected light.

The effective temperatures of externally heated extrasolar planets can be estimated from energy balance as

$$
T_{p}=T_{*}\left(\frac{R_{*}}{a}\right)^{1 / 2}\left[f\left(1-A_{\mathrm{B}}\right)\right]^{1 / 4},
$$

where $T_{p}$ is the temperature of the planet, $T_{*}$ and $R_{*}$ are the effective temperature and the radius of the star, $a$ is the star-planet orbital separation, and $f$ and $A_{\mathrm{B}}$ are the re-radiation factor and the Bond albedo of the planet.

Given the planets effective temperatures, their thermal and reflected fluxes can be computed using

$$
F_{p_{t h}}=\frac{2 h \nu^{3}}{c^{2}} \frac{\pi R_{p}^{2}}{e^{\frac{h \nu}{k T_{p}}}-1} \frac{1}{D^{2}},
$$

for the thermal flux, and

$$
F_{p_{r e f}}=F_{*} A_{g} \frac{R_{p}^{2}}{a^{2}}
$$


for the reflected flux, where $F_{*}$ is the stellar surface flux divided by $D^{2}, A_{g}=\frac{2}{3} A_{B}$, assuming Lambert's law (e.g., Rowe et al. 2006), and $R_{p}$ is the radius of the planet. $D$ is the distance from the planet to Earth.

\subsection{Effective Temperatures}

We computed temperatures for each known VHJ from eq. (1), adopting published masses, radii, and effective temperatures for the host stars (see Table 1). The orbital separations were derived from the mass of the stars and the planets, and the published orbital periods.

The parameter $f$ describes how the stellar radiation absorbed by a planet is redistributed in its atmosphere. The absorbed radiation can be reradiated back to space, advected around the planet, or a combination of both. If the radiative timescale is shorter than the advective timescale, the incident radiation will be reradiated back to space, in which case $f=2 / 3$. In the opposite case, atmospheric circulation redistributes the energy around the planetary atmosphere before it can get reradiated, and $f=1 / 4$. Recent studies of the day-to-night side brightness variations of $v$ Andromedae b (Harrington et al. 2006), and HD189733b (Knutson et al. 2007), indicate that both scenarios are possible. We consider values of $f=$ $2 / 3-1 / 4$ to bracket the possible range.

The Bond albedo $A_{B}$ is the fraction of incident stellar radiation reflected by the planets atmosphere. $A_{B}$ depends on a planet's chemical composition, which in turn depends partly on temperature. We consider the cases $A_{B}=0,0.3$, and 0.5. Observations indicate that the albedos of hot Jupiters are in fact very low $\left(A_{B}<0.15\right.$; Rowe et al. 2006; Harrington, priv. comm.). Model atmospheres also suggest these planets are dark (e.g. Marley et al. 2007). Even so, we include $A_{B}=0.3$ and 0.5 to illustrate possible unexpectedly reflective cases. VHJs may be as bright as $A_{B}=0.3$ if covered by homogeneous pure silicate clouds at one millibar level or higher altitudes (Marley et al. 1999, Seager et al. 2000, Sudarsky et al. 2000). If they have patchy silicate or iron clouds, then $A_{B}$ will be $<0.3$, as the stellar radiation can penetrate below the clouds and be absorbed by gas-phase molecules (Hood et al. 2007; submitted). Of most relevance to this work is that VHJ atmospheres are too hot on the substellar side for silicate or iron clouds to form.

Table 1 gives the expected $T_{p}$ for each known transiting VHJ, for the $f$ and $A_{B}$ values above. The table includes formal errors derived from eq. 1 and published parameters of each system. The maximum expected temperatures in the table reveal two groups of VHJs. One

with $T_{p_{M a x}}>2600 \mathrm{~K}$, that includes OGLE-TR-56b and OGLE-TR-132b, and a second group 
with $T_{p_{M a x}}<2200 \mathrm{~K}$, that includes the other nine planets. OGLE-TR-56b and OGLE-TR$132 \mathrm{~b}$ are the most likely candidates for detection.

\subsection{Thermal Emission and Reflected Light Fluxes}

A successful detection of radiation from transiting extrasolar planets depends on the ratio of fluxes emitted by the planet and the star at a given wavelength. Figure 1 shows the computed model stellar fluxes for OGLE-TR-56 and OGLE-TR-132, between 0.5 and $2.5 \mu \mathrm{m}$, and their expected thermal emission and reflected light fluxes, based on the $T_{p}$ and $A_{B}$ values from $\S 2.1$.

The stellar fluxes are derived using grids of Kurucz (1993) models, interpolated for the specific $T_{\text {eff }}$, [Fe/H], and $\log g$ values of OGLE-TR-56 and OGLE-TR-132. Stellar parameters for OGLE-TR-56 have been measured by Santos et al. (2006), who obtain $T_{\text {eff }}=6119 \pm$ $62 \mathrm{~K},[\mathrm{Fe} / \mathrm{H}]=+0.25 \pm 0.08 \mathrm{dex}$, and $\log g=4.21 \pm 0.19 \mathrm{cgs}$. For OGLE-TR-132, the stellar parameters derived by Bouchy et al. (2004) are $T_{\text {eff }}=6411 \pm 179 \mathrm{~K},[\mathrm{Fe} / \mathrm{H}]=+0.43 \pm 0.18$ dex, and $\log g=4.86 \pm 0.14 \mathrm{cgs}$.

For the planets, we derive thermal emission and reflected light fluxes from eqs. (2) and (3), assuming the planets and the stars emit as blackbodies. We consider two temperature scenarios: 1) $f=1 / 4$ and $A_{B}=0.5$, where the planetary fluxes in the optical are dominated by reflected light (such high albedos are unlikely for VHJs, see $\S 2.1$ ), and 2) $f=2 / 3$ and $A_{B}=0.05$, close to the $f=2 / 3 ; A_{B}=0.0$ scenario, to illustrate the significantly lower contribution of reflected light versus thermal emission for very low albedos. The expected temperatures of OGLE-TR-56b and OGLE-TR-132b in scenario 2) are $2852 \pm 24 \mathrm{~K}$ and 2581 $\pm 35 \mathrm{~K}$.

We go beyond blackbody models by including in Fig. 1 the Hubeny et al. (2003) models for irradiated planets at $T_{p}=2600 \mathrm{~K}$, with and without $\mathrm{TiO} / \mathrm{VO}$ molecules in their atmospheres. In the latter case, $\mathrm{TiO}$ and $\mathrm{VO}$ have condensed into solids, no longer contributing to the opacity. $\mathrm{TiO}$ and $\mathrm{VO}$ are such strong absorbers that the incident stellar radiation is absorbed at an altitude where reradiation dominates over advection, potentially leading to very hot planetary atmospheres on the substellar side. The presence of $\mathrm{TiO}$ and $\mathrm{VO}$ is furthermore expected to create a temperature inversion in the lower atmosphere in some cases (e.g., metal rich, see also Fortney et al. 2006), leading to emission lines and making the planet flux at some wavelengths brighter than blackbodies of the same temperature. 


\section{Detectability of Secondary Transits at Optical Wavelengths}

Secondary transits can be detected at any given wavelength if the planet-to-star flux ratios, $F_{p} / F_{*}$, are high enough. In terms of magnitudes, the transits are detectable if the difference in magnitude during transit, $\mid \Delta$ mag $\mid$, is larger than the photometric precision of the observational light curves, $\sigma_{\text {mag }} . \mid \Delta$ mag $\mid$ is derived as

$$
|\Delta m a g|=2.5 \cdot \log _{10}\left(1+\frac{F_{p}}{F_{*}}\right),
$$

where $F_{p}$ is either the thermal emission flux, $F_{p_{t h}}$, or the reflected light flux, $F_{p_{r e f}}$. Detectability can be further increased by binning the transit data, as done for example by Deming et al. (2005) for HD209458b. The photometric precision of the light curves is then $\sigma_{\text {mag }} / \sqrt{t}$, where $t$ is the time duration of the bins.

Figure 2 shows the expected $\mid \Delta$ mag $\mid$ during secondary transits of OGLE-TR-56b and OGLE-TR-132b, for the thermal emission and reflected light cases in Fig. 1. A detailed description is given in the figure's caption. The stellar and planetary fluxes have been integrated over two model filter passbands with FWHMs $=0.1 \mu \mathrm{m}$, centered at $0.76 \mu \mathrm{m}$ and $0.91 \mu \mathrm{m}$. Those passbands resemble the $i^{\prime}$ - and $z^{\prime}$-band filters of the Sloan Digital Sky Survey (SDSS; Fukugita et al. 1996).

We also show the achievable $\sigma_{m a g}$ for OGLE transit light curves at optical wavelengths (horizontal dashed lines in Fig. 2) for integration times of $t=1,120(3 \sigma)$, and $120(1 \sigma)$ minutes. The transits of both planets last about $120 \mathrm{~min}$, so the two bottom lines represent the $1 \sigma$ and $3 \sigma$ level photometric precision that would be achieved by binning $\sigma_{m a g}=1.0$ mmags/min light curve data over the entire duration of the transits (see below and $\S 4$ ).

To compare our estimates with $\sigma_{m a g}$ from OGLE primary transit light curves, we give the average $\sigma_{m a g}$ and exposure times per data point in Table 2. Assuming no correlation between photometric precision and filter passbands, $\sigma_{\text {mag }}$ varies between 0.83 and $1.18 \mathrm{mmags} / \mathrm{min}$, depending on the instrument and the target. Here we have excluded the Magellan/IMACS values because its low photometric precision results from instrumental effects (Winn et al. 2007b). We therefore reasonably assume an average $\sigma_{m a g}$ of $1.0 \mathrm{mmags} / \mathrm{min}$.

\section{Discussion}

Secondary transits of extrasolar planets are normally pursued in the infrared, where $F_{p_{t h}} / F_{*}$ is higher than in the optical. We argue that, for the right combination of stellar and planetary parameters, the thermal emission from some VHJs could be detected at optical 
wavelengths during secondary transits.

Our investigation of all known transiting VHJs concludes that only two of them, OGLETR-56b and OGLE-TR-132b, have the right combination of stellar and planetary parameters to be hotter than $2600 \mathrm{~K}$. At those temperatures thermal emission dominates over reflected light emission. Therefore thermal emission could be detected during secondary transits in the optical. OGLE-TR-56b is the most promising candidate for detection, in either $i^{\prime}-$ or $z^{\prime}$-band. OGLE-TR-132b could also be detected in $z^{\prime}$-band, but the prospects are lower. Reflected light emission is similar in both filters, and a factor of 10-20 less than the thermal emission if the planets are very hot.

Observations in $z^{\prime}$-band are better suited for detecting thermal emission from these two planets than ground-based observations at other optical or near-IR passbands. The better performance of $z^{\prime}$-band versus $i^{\prime}$-band is clearly illustrated in Fig. 2. At shorter wavelengths, e.g. $0.50-0.65 \mu \mathrm{m}, F_{p} / F_{\text {st }}$ decreases considerably (see Fig. 1). At near-IR wavelengths, the expected $F_{p} / F_{s t}$ are higher, but the effect of the atmosphere on ground-based observations produce lower quality light curves (Snellen \& Covino 2007; Díaz et al. 2007).

The transits of OGLE-TR-56b and OGLE-TR-132b last for about two hours. By binning $z^{\prime}$-band light curves with $\sigma_{m a g}=1.0 \mathrm{mmag} / \mathrm{min}$ over the duration of the transits (in-transit and out-of-transit bins of the same duration), thermal emission from OGLE-TR-56b could be detected with $>5 \sigma$ significance after three secondaries, if the planet emits as a blackbody with $T_{p}>2500 \mathrm{~K}$. If the planet emits as predicted by the Hubeny et al. (2003) models with $\mathrm{TiO} / \mathrm{VO}$, the expected transit depth is $0.04 \%$ (filled magenta squares in Fig. 2). The transit could be then detected with $>6 \sigma$ significance after three transits. In the case of OGLE-TR-132b, four secondary transits are needed for a $5 \sigma$ significance detection.

One might expect that cooler VHJs around brighter stars would be as promising or more so than the more distant and fainter (yet hotter) OGLE-TR-56b and OGLE-TR-132b. In principle, slightly cooler planets around brighter stars should be just as favorable, because the brighter stars have lower Poisson photon noise. In practice, $\sigma_{m a g}$ is limited for bright stars in two ways. On small telescopes atmospheric scintillation limits $\sigma_{m a g}$ from reaching the photon noise limit ( $\sigma_{\text {mag }}$ from scintillation is typically several millimags). On large telescopes the limit is usually imposed by the absence of suitable nearby comparison stars within their small fields of view.

Any newly discovered transiting VHJs should be examined for the possibility of detecting their thermal emission at optical wavelengths. In particular hot VHJs orbiting bright stars with a binary star companion would be ideal. Such detections will provide important clues about the energy processing mechanisms undergoing in the atmospheres of those planets. 
M. L-M. acknowledges support from the Carnegie Institution of Washington through a Carnegie Fellowship, and from the NASA Astrobiology Institute. We thank A. Bonanos for

her help generating Kurucz models and I. Hubeny for helpful clarifications on his models. We also thank an anonymous referee for helpful suggestions.

\section{REFERENCES}

Ballester, G. E., Sing, D. K. \& Herbert, F. 2007, Nature, 445, 511

Bouchy, F., Pont, F., Santos, N. C. et al. 2004, A\&A, 421, L13

Bouchy, F., Udry, S., Mayor, M. et al. 2005, A\&A, 444, L15

Burke, C. J., McCullough, P. R., Valenti, J. A. et al. 2007, arXiv:0705.0003

Charbonneau, D., Brown, T. M., Noyes, R. W. \& Gilliland, R. L. 2002, ApJ, 568, 377

Charbonneau, D., Allen, L. E. Megeath, S. T. et al. 2005, ApJ, 626, 523

Charbonneau, D., Winn, J. N., Everett, M. E. et al. 2007, ApJ, 658, 1322

Díaz, R. F., Ramírez, S., Fernández, J. M. et al. 2007, ApJ, 660, 850

Deming, D., Seager, S., Richardson, L. J. \& Harrington, J. 2005, Nature, 434, 740

Deming, D., Harrington, J., Seager, S. \& Richardson, L. J. 2006, ApJ, 644, 560

Deming, D., Richardson, L. J. \& Harrington, J. 2007, MNRAS, in press

Fortney, J. J., Saumon, D., Marley, M. S. et al. 2006, ApJ, 642, 495

Fukugita, M., Ichikawa, T., Gunn, J. E. et al. 1996, AJ, 111, 1748

Gillon, M., Pont, F., Moutou, C. et al. 2006, A\&A, 459, 249

Grillmair, C. J., Charbonneau, D., Burrows, A. et al. 2007, ApJ, 658, L115

Harrington, J. Hansen, B. M., Luszcz, S. H. et al. 2006, Science, 314, 623

Holman, M. J., Winn, J. N., Fuentes, C. I. et al. 2007, ApJ, 655, 1103

Hubeny, I. Burrows, A. \& Sudarsky, D. 2003, ApJ, 594, 1011

Knutson, H. A., Charbonneau, D., Allen, L. E. et al. 2007, arXiv:0705.0993 
Kurucz, R. 1993, CD-ROM 13, ATLAS9 Stellar Atmosphere Programs and 2 km/s Grid (Cambridge: SAO)

Marley, M. S., Fortney, J. J., Hubickyj, O. et al. 2007, ApJ, 655, 541

Marley, M. S., Gelino, C., Stephens, D. et al. 1999, ApJ, 513, 879

Moutou, C., Pont, F., Bouchy, F. \& Mayor, M. 2004, A\&A, 424, 31

O’Donovan, F. T., Charbonneau, D., Bakos, G. et al. 2007, arXiv:0705.2004

Pont, F., Moutou, C., Gillon, M. et al. 2007, A\&A, 465, 1069

Richardson, L. J., Deming, D., Horning, K. et al. 2007, Nature, 445, 892

Rowe, J. F., Matthews, J. M., Seager, S. et al. 2006. ApJ, 646, 1241

Santos, N. C., Pont, F., Melo, C. et al. 2006, A\&A, 450, 825

Sato, B., Fischer, D. A., Henry, G. W. et al 2005, ApJ, 633, 465

Seager, S., Whitney, B. A.\& Sasselov, D. D. 2000, ApJ, 540, 504

Snellen, I. A. G. 2005, MNRAS, 363, 211

Snellen, I. A. G. \& Covino, E. 2007, MNRAS, 375, 307

Sozzetti, A., Torres, G., Charbonneau, D. et al. 2007, arXiv:0704.2938

Sudarsky, D., Burrows, A. \& Pinto, P. 2000, ApJ, 538, 885

Tinetti, G., Vidal-Madjar, A., Liang, M-C. et al. 2007, Nature, 448, 169

Vidal-Madjar, A., Lecavelier des Etangs, A., Désert, J.-M. et al.2003, Nature, 422, 143

Vidal-Madjar, A., Désert, J.-M., Lecavelier des Etangs, A. et al. 2004, ApJ, 604, L69

Winn, J. N., Holman, M. J., Henry, G. W. et al. 2007a, AJ, 133, 1828

Winn, J. N., Holman, M. J. \& Fuentes C. I. 2007b, AJ, 133, 11

This preprint was prepared with the AAS IATEX macros v5.2. 
Table 1: Effective temperatures of the eleven known transiting VHJs, for $A_{B}=0.0,0.3$, and 0.5 , and $f=2 / 3$ and $1 / 4$.

\begin{tabular}{|c|c|c|c|c|c|c|c|}
\hline Planet & $\begin{array}{c}T_{p}(K) \\
\mathrm{f}=2 / 3 ; A_{B}=0\end{array}$ & $\begin{array}{c}T_{p}(K) \\
\mathrm{f}=2 / 3 ; A_{B}=0.3\end{array}$ & $\begin{array}{c}T_{p}(K) \\
\mathrm{f}=2 / 3 ; A_{B}=0.5\end{array}$ & $\begin{array}{c}T_{p}(K) \\
\mathrm{f}=1 / 4 ; A_{B}=0\end{array}$ & $\begin{array}{c}T_{p}(K) \\
\mathrm{f}=1 / 4 ; A_{B}=0.3\end{array}$ & $\begin{array}{c}T_{p}(K) \\
\mathrm{f}=1 / 4 ; A_{B}=0.5\end{array}$ & Ref. $^{1}$ \\
\hline OGLE-TR-56b & $2889 \pm 24$ & $2642 \pm 22$ & $2429 \pm 20$ & $2260 \pm 19$ & $2068 \pm 17$ & $1901 \pm 16$ & 1,2 \\
\hline OGLE-TR-113b & $1717 \pm 14$ & $1570 \pm 13$ & $1444 \pm 12$ & $1344 \pm 11$ & $1229 \pm 10$ & $1130 \pm 9$ & $1,3,4$ \\
\hline OGLE-TR-132b & $2615 \pm 36$ & $2392 \pm 32$ & $2199 \pm 30$ & $2046 \pm 28$ & $1872 \pm 25$ & $1721 \pm 23$ & 5,6 \\
\hline HD189733b & $1500 \pm 10$ & $1372 \pm 9$ & $1261 \pm 8$ & $1174 \pm 8$ & $1074 \pm 7$ & $987 \pm 6$ & 7,8 \\
\hline $\mathrm{XO}-2 \mathrm{~b}$ & $1682 \pm 15$ & $1539 \pm 14$ & $1415 \pm 13$ & $1316 \pm 12$ & $1204 \pm 11$ & $1107 \pm 10$ & 9 \\
\hline Corot-exo-1b & 2225 & 2036 & 1871 & 1742 & 1593 & 1464 & 10 \\
\hline WASP-1 & $2177 \pm 61$ & $1991 \pm 56$ & $1831 \pm 51$ & $1704 \pm 48$ & $1558 \pm 44$ & $1433 \pm 40$ & 11 \\
\hline WASP-2 & $1615 \pm 96$ & $1478 \pm 87$ & $1358 \pm 80$ & $1264 \pm 75$ & $1156 \pm 68$ & $1063 \pm 63$ & 11 \\
\hline HD140926b & $2226 \pm 30$ & $2036 \pm 27$ & $1872 \pm 25$ & $1742 \pm 23$ & $1593 \pm 21$ & $1465 \pm 20$ & 12 \\
\hline TrES-2 & $1882 \pm 15$ & $1722 \pm 14$ & $1583 \pm 13$ & $1473 \pm 12$ & $1347 \pm 11$ & $1238 \pm 10$ & 13 \\
\hline TrES-3 & $2100 \pm 32$ & $1921 \pm 29$ & $1766 \pm 27$ & $1643 \pm 25$ & $1503 \pm 23$ & $1382 \pm 21$ & 14 \\
\hline
\end{tabular}

${ }^{1}$ [1] Santos et al. (2006), [2] Pont et al. (2007), [3] Gillon et al. (2006), [4] Díaz et al. (2007), [5] Bouchy et al. (2004), [6] Moutou et al. (2004), [7] Bouchy et al. (2005), [8] Winn et al. (2007a), [9] Burke et al. (2007), [10] European Space Agency (ESA) press release, [11] Charbonneau et al. 2007, [12] Sato et al. (2005), [13] Sozzetti et al. (2007), O'Donovan et al. (2007). 
Table 2: Parameters of the best published optical light curves of OGLE transiting planets.

\begin{tabular}{rccccl}
\hline \hline Planet & $\begin{array}{c}\text { Central } \lambda \\
(\AA)\end{array}$ & Telescope/Instr. & $\begin{array}{c}\text { Light Curve Dispersion } \\
(\mathrm{mmags})\end{array}$ & $\begin{array}{c}\text { Exposure Time } \\
(\mathrm{sec})\end{array}$ & Ref. Source \\
\hline OGLE-TR-132b & 6550 & ESO VLT/FORS2 & 1.2 & $55-60$ & Moutou et al. (2004) \\
OGLE-TR-10b & 8500 & Magellan/MagIC & 0.77 & $55-85$ & Holman et al. (2007) \\
OGLE-TR-111b & 8100 & Magellan/IMACS ${ }_{-}^{3}$ & $1.5-2.0$ & $75-125$ & Winn et al. (2007b) \\
OGLE-TR-113b & 6400 & ESO NTT/SUSI2 & $1.2-1.26$ & 55 & Gillon et al. (2006) \\
OGLE-TR-56b & 6550 & ESO VLT/FORS1 & 0.9 & $60^{-5}$ & Pont et al. (2007) \\
\hline \hline
\end{tabular}




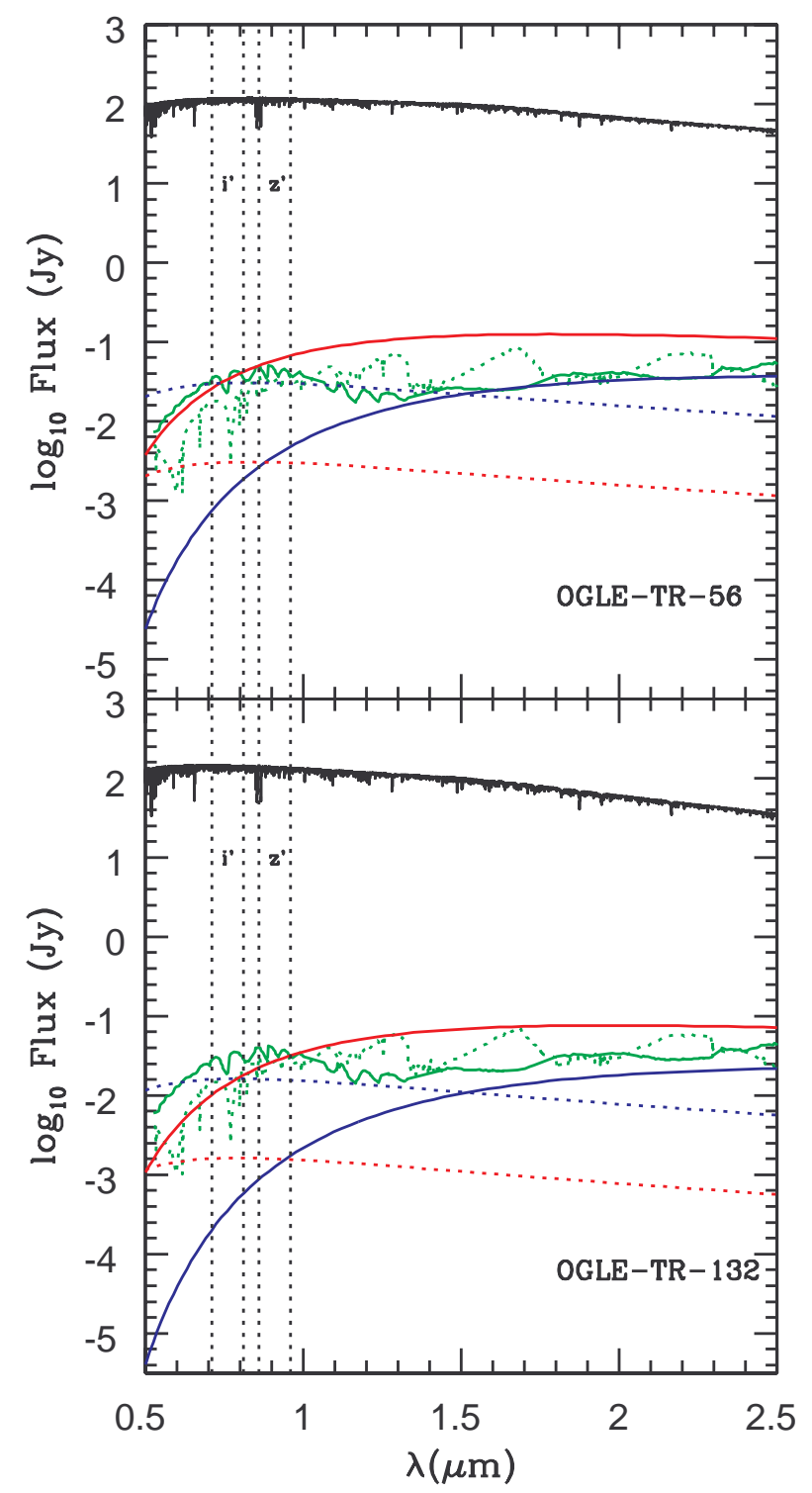

Fig. 1.- Model stellar fluxes for OGLE-TR-56A-b (top) and OGLE-TR-132A-b (bottom) between 0.5 and $2.5 \mu \mathrm{m}$. Black lines represent Kurucz (1993) models for the stars, multiplied by $4 \pi$ and $R_{s t}^{2} / D^{2}$. Green lines show the $T_{p}=2600 \mathrm{~K}$ Hubeny et al. (2003) models with (solid line) and without (dotted line) $\mathrm{TiO} / \mathrm{VO}$. These models are multiplied by $4 \pi$ and $R_{p}^{2} / D^{2}$. The red and blue solid lines are the planets' blackbody thermal emission for $f=2 / 3 ; A_{B}=0.05$, and $f=1 / 4 ; A_{B}=0.5$ (see $\S 2.2$ ). The red and blue dotted lines are reflected light emission for the same two cases. All the fluxes are normalized to a distance $D=10 \mathrm{pc}$. $D$ is only a normalization factor and has no effect on the results in Fig. 2. For very low albedos $A_{B}$ and inefficient energy redistribution factors $f$, the thermal emission of the planets dominates over their reflected light emission. The two vertical columns (dotted lines) show the $i^{\prime}$ and $z^{\prime}$ model passbands described in $\S 3$. 

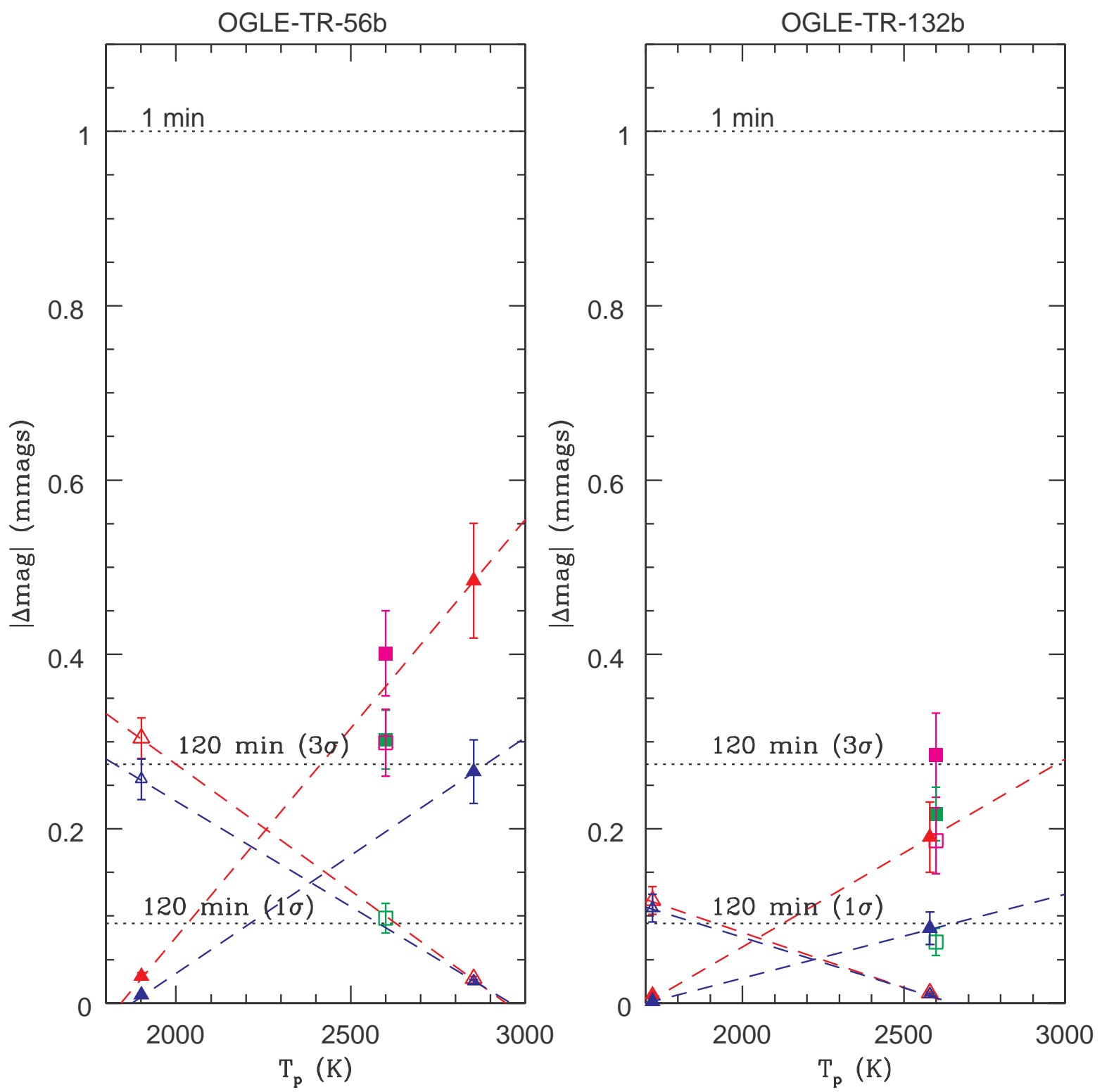

Fig. 2.- Expected depths of secondary transits, $|\Delta \mathrm{mag}|$, as a function of $T_{p}$ for OGLE-TR56b (left-side) and OGLE-TR-132b (right-side). Filled triangles show the expected $|\Delta \mathrm{mag}|$ if the planets emit thermally as blackbodies (see Fig. 1). Open triangles correspond to the cases of reflected light. The dashed lines connecting the points guide the eye on how the value of $\mid \Delta$ mag $\mid$ varies with the temperature of the planet $T_{p}$ in each case. Red illustrates the results in $z^{\prime}$-band; blue correspond to $i^{\prime}$-band. Green and magenta squares indicate the values of $|\Delta \mathrm{mag}|$ if the planets emit as predicted by the Hubeny et al. (2003) models. Green shows the results in $i^{\prime}$-band and magenta in $z^{\prime}$-band. Filled and open symbols in this case show the results the models with and without $\mathrm{TiO} / \mathrm{VO}$. The errorbars on each point have been estimated by formal propagation of errors. Finally, the horizontal dotted lines indicate the photometric precision achievable with current instruments on ground-based telescopes in $\mathrm{t}=1,120(3 \sigma)$, and $120(1 \sigma)$ minutes. 